\title{
A Novel Time and Voltage Based SAR ADC Design With Self-Learning Technique
}

\author{
Abhilash Karnatakam Nagabhushana \\ Dept. of Electrical and Computer Engineering \\ Southern Illinois University \\ Carbondale USA \\ kn.abhilash6@siu.edu
}

\author{
Haibo Wang \\ Dept. of Electrical and Computer Engineering \\ Southern Illinois University \\ Carbondale, USA \\ haibo@engr.siu.edu
}

\begin{abstract}
This paper presents a novel time and voltage based technique for successive approximation register (SAR) analogto-digital converter (ADC) to improve the conversion speed. By taking advantage of the fact that at low supply voltage there will be a significant difference in comparator decision time for different input voltages, the proposed technique creates multiple auxiliary voltage levels for comparison and hence eliminates the need of additional comparators for acceleration as compared with the existing methods. In addition, a digital self-learning module is also presented, which calculates the uncertainty window required for bound update in the proposed method and thus adjusts to different process corners. To validate these concepts, a 10-bit SAR ADC is designed in $130 \mathrm{~nm}$ CMOS process with $0.5 \mathrm{~V}$ power supply voltage. The circuit operates in both conventional and proposed modes. Simulations show that the largest number of conversion cycles is 7 , hence resulting in an acceleration of $30 \%$ over the conventional scheme, while the average number of cycles is 5.58. Simulation results also demonstrate that the proposed method does not affect accuracy. Both ADC operation modes achieve SNDR (signal-tonoise distortion ratio) of $59 \mathrm{~dB}$, corresponding to an ENOB (effective number of bits) of 9.5-bits.
\end{abstract}

\section{Keywords}

SAR ADC, low-voltage, self-learning

\section{INTRODUCTION}

SAR ADC is an attractive topology for implementing lowvoltage data converter circuits in the nano scale technology nodes, thanks to its simple structure and not requiring highgain amplifiers for residual amplification. Recently, there are quite a few low-power or high-performance SAR ADC circuits reported in literature [1-10]. Typically, SAR ADCs implement the binary search algorithm and require $\mathrm{N}$ conversion cycles to generate an N-bit digital output. Additionally, with the scaling down of power supply, it takes longer time

Permission to make digital or hard copies of all or part of this work for personal or classroom use is granted without fee provided that copies are not made or distributed for profit or commercial advantage and that copies bear this notice and the full citation on the first page. Copyrights for components of this work owned by others than ACM must be honored. Abstracting with credit is permitted. To copy otherwise, or republish, to post on servers or to redistribute to lists, requires prior specific permission and/or a fee. Request permissions from permissions@acm.org.

DAC '16, June 05-09, 2016, Austin, TX, USA

(c) 2016 ACM. ISBN 978-1-4503-4236-0/16/06 . . $\$ 15.00$

DOI: http://dx.doi.org/10.1145/2897937.2897970 for charge scaling (CS) capacitor arrays to settle and the comparator decision time also increases. All these factors adversely affect low-voltage SAR ADC conversion speed. In the past various techniques had been developed to address this challenge from different aspects.

Multi-bits per cycle techniques have been used to reduce the total number of conversion cycles [1-5]. This is achieved by simultaneously comparing the ADC input with multiple levels by using multiple comparators. To keep the hardware overhead reasonable, the number of bits to be generated in a single cycle is typically 2 or less. Nevertheless, the additional hardware required in these designs is still significant. An interesting technique [5] has been presented to reduce the number of comparators; but it negatively affects the circuit speed. To relax the CS array settling requirement, redundancy can be embedded into the search process either by using non-binary search methods [6] or introducing additional compensation steps [7]. The redundancy enables the ADCs to reach the correct digital output code even if there is large CS capacitor array settling errors in the early conversion cycles. This allows short time durations to be budgeted for CS capacitor array settling. Thus, the overall ADC conversion time can be reduced, despite that extra conversion cycles have to be performed in these redundancy based ADC circuits.

With the scaling down of power supply voltage, the comparator resolving time, the duration taken by the comparator to make a valid decision, increases exponentially as shown in Fig. 1. Two factors contribute to such rapid increase of comparator resolving time. One is the reduced device transconductance in low-voltage operation and the other is the decreasing ADC $V_{L S B}$, which represents the minimum

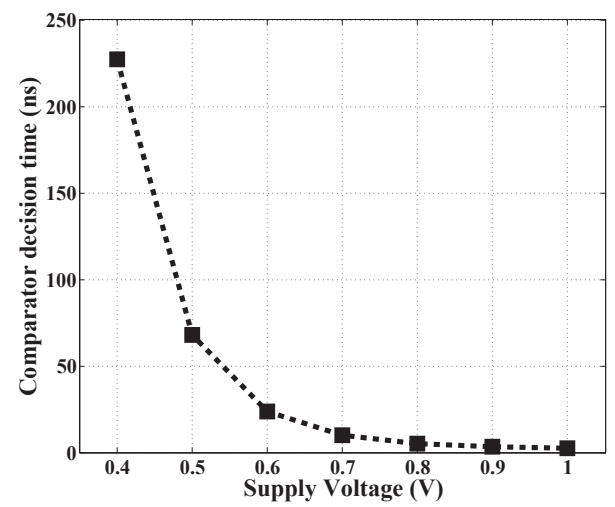

Figure 1: Comparator decision time for different Supply Voltages 
voltage difference to be detected by the comparator. Although the increased comparator resolving time is generally regarded as an adverse factor in ADC design, it also provides an opportunity to extract additional information from comparator transient behavior. Several recent ADC circuits exploit this opportunity in different ways. The designs in $[8,9]$ take advantage of timing information to detect comparator metastability, subsequently improving ADC resolution or accelerating the conversion process. The proximity detector in [9] also relies on comparator timing information to detect when noise signal can be injected for background calibration. According to comparator timing information, the ternary SAR ADC in [10] divides the search space into three sub regions similar to that in pipelined ADCs with 1.5-bits per stage. Overall, these novel designs indicate the promising potentials of utilizing comparator timing information in SAR ADC design. However, bit information extracted from comparator transient responses remains limited in the existing designs.

This paper presents a novel technique that is based on the fundamental fact that, the smaller the differential input voltage is, the longer the time taken by the comparator to make an output decision and this is especially true if we have a low supply voltage. This motivates us to present a time and voltage bound based search algorithm that creates several auxiliary voltage levels for comparison using the relation between the decision time and differential input voltage of a voltage based comparator circuit. Also, a self-learning digital module is presented, which makes the circuit process independent by adjusting itself to work under all the corners.

The proposed ADC technique is explained in Section 2. In Section 3 circuit implementation and its problems are discussed. Simulation results to validate the proposed techniques are provided in Section 4 and finally conclusions are drawn in Section 5.

\section{PROPOSED TIME AND VOLTAGE BASED SAR METHOD}

Fig. 2 shows the time taken by the comparator to resolve the differential input voltage for a power supply of $0.5 \mathrm{~V}$. This information is a key component in our design. It can be observed from the plot that as the differential input voltage increases the decision time of the comparator reduces quickly. The above phenomenon is not significant with high supply voltages because of large transistor transconductance in such circuits but it is more dramatic for low supply volt-

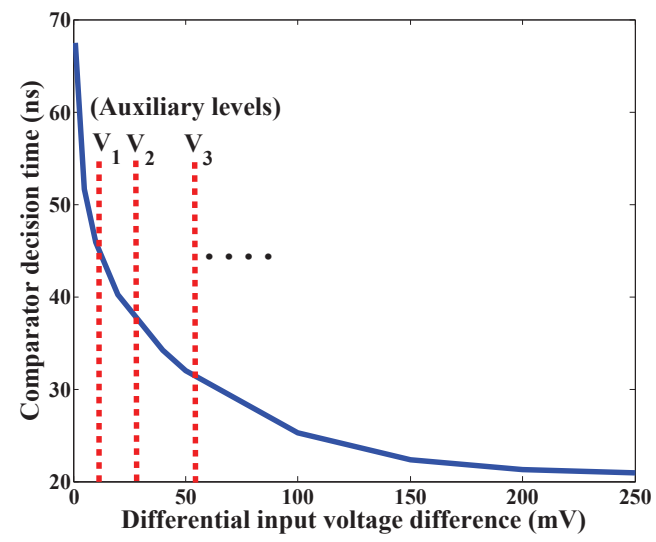

Figure 2: Comparator decision time
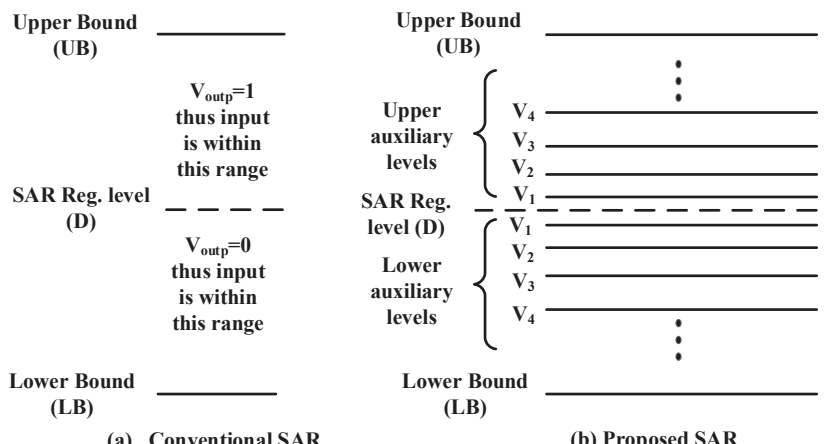

(a) Conventional SAR

(b) Proposed SAR

Figure 3: Conventional and Proposed SAR search space

age implementations. Also, if the clock frequency is high, comparator cannot make a legitimate decision. Hence the comparator should be given enough time to resolve the input difference. Based on these observations, we can select multiple auxiliary voltage levels and create a level based search scheme. Care should be taken in selecting these auxiliary voltage levels such that there is a significant difference between its corresponding time levels too. Also, the decision time roughly becomes same if the differential input is more than $100 \mathrm{mV}$. So, the additional levels that are to be selected need to be less than or equal to $100 \mathrm{mV}$.

Therefore, the input signal can be easily categorized into its respective voltage levels based on the decision time of comparator. The search space for the conventional SAR and the proposed method are shown in Fig. 3. The conventional scheme uses the binary search process and hence reduces the search space by half in every cycle, while in the proposed scheme the amount of acceleration achieved can be higher because the input is categorized and the search space is updated based on auxiliary voltage levels.

Apart from these things it is also important to investigate the impact on comparator decision time under different process corners, which is illustrated in Fig. 4. It can be clearly seen that decision time is different at different process corners, under such circumstances the above described method fails to work. A solution to the above case is to find a delay element that can actually mimic the comparator. The first choice of delay element is a buffer, whose propagation delay is also subjected to vary under different corners. It is observed that, if we normalize the comparator decision time to an unit sized buffer delay the curves fail to overlap and are

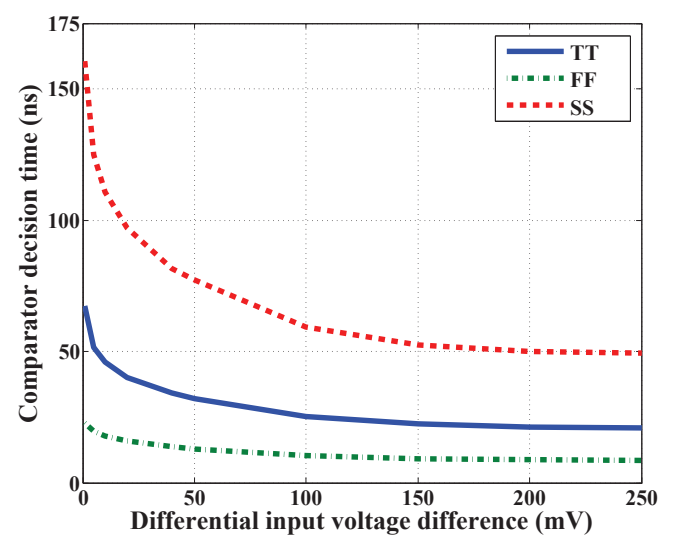

Figure 4: Comparator decision time for all corners 


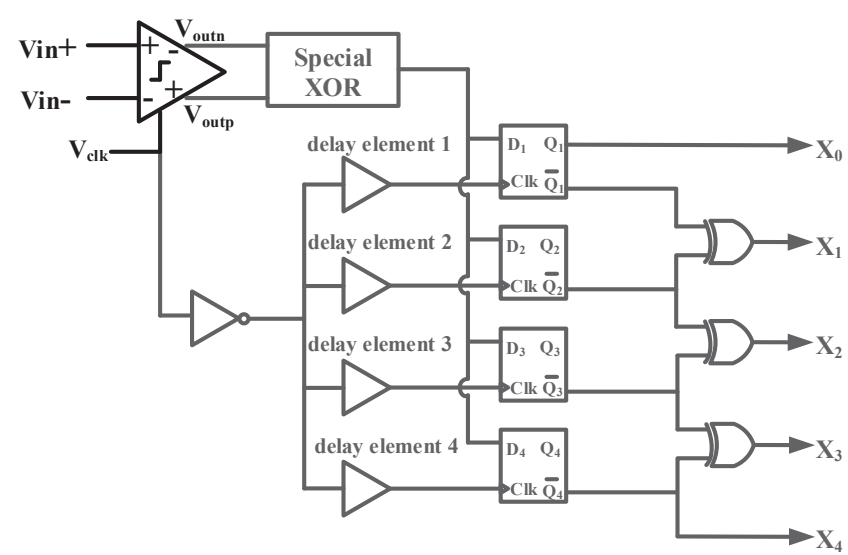

Figure 5: Setup to extract comparator decision time information

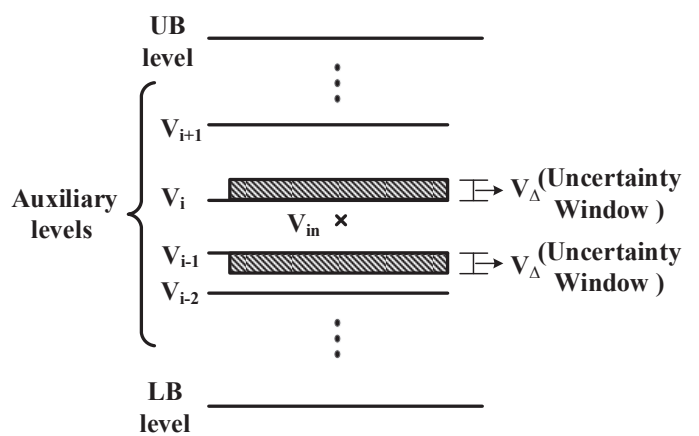

Figure 6: Bound update

widely apart. Hence, buffer is not a suitable delay element and we need to look for alternative designs. Theoretically, if we implement a delay element in the same fashion as the comparator it is possible that curves might overlap close enough. However, this also leads to an uncertainty in the selection of voltage levels as they cannot be the same for all the corners due to comparator offset. So, this uncertainty window has to be counted and it will be addressed during the bound update procedure to be discussed later.

Fig. 5 shows the setup to extract the comparator decision time information. It consists of a special XOR gate connected to the comparator outputs ( $V_{\text {outp }}$ and $\left.V_{\text {outn }}\right)$, and it goes to logic 0 whenever comparator makes a decision. The comparator is reset when the clock is high and goes to regeneration or decision mode when clock is low. A series of D flip-flops (DFFs) are connected to this XOR gate output to capture its transition and they are triggered by different clocks. The XOR gate is designed to have sharp output transitions thus minimizing metastability in DFFs. Special delay elements similar to comparator are used to derive the clocks for DFFs from the comparator clock. These delays are obtained corresponding to multiple auxiliary voltage levels

Table 1: Comparator decision time information

\begin{tabular}{|c|c|c|}
\hline Differential input $V_{i n}$ & $\overline{Q_{4}} \overline{Q_{3}} \overline{Q_{2}} \overline{Q_{1}}$ & $X_{4} X_{3} X_{2} X_{1} X_{0}$ \\
\hline \hline $0<V_{i n}<V_{1}$ & 0000 & 00001 \\
\hline$V_{1}<V_{i n}<V_{2}$ & 0001 & 00010 \\
\hline$V_{2}<V_{i n}<V_{3}$ & 0011 & 00100 \\
\hline$V_{3}<V_{i n}<V_{4}$ & 0111 & 01000 \\
\hline$V_{4}<V_{i n}$ & 1111 & 10000 \\
\hline
\end{tabular}

selected from Fig. 2. Note, in the proposed design we have 4 auxiliary levels in each half of the search space. The possible output pattern of these DFFs $\left(\bar{Q}_{1} \bar{Q}_{2} \bar{Q}_{3} \bar{Q}_{4}\right)$ and the corresponding differential inputs are listed in Table 1. Since for the decision making process we just want one of their outputs to be high, we add additional normal XOR gates at the output of these DFFs. Among outputs $X_{0}, X_{1}, X_{2}, X_{3}$ and $X_{4}$ only one of them will be high at any time after the comparator decision.

Based on this, we know where the input lies between which voltage levels and we proceed for bound update. Once the bounds are updated to the lowest auxiliary levels, acceleration is no longer possible and the normal search process is followed. Since the comparator results are prone to temperature and process variations we need to add an uncertainty window for safe update of bounds without losing information as depicted in Fig. 6. This uncertainty window can be variable here because of the fact that comparator takes longer time to make a decision for smaller input voltage difference, accordingly this uncertainty window can be small at the initial auxiliary levels and can be large at later auxiliary levels.

Considering all these things the lower bound (LB) and upper bound (UB) update policy is described as shown in Fig. 7. where, D, $V_{i, d i g}$ and $\Delta$ are digital codes for SAR register level, auxiliary level and uncertainty window respectively. In both these updates $V_{i, d i g}+\Delta$ and $V_{i-1, d i g}-\Delta$ remain common, so it is easy to generate these values. Note that $\Delta$ can be variable and this aids in better acceleration as compared to constant $\Delta$. The problem with uncertainty window $\Delta$ is that it has to be calculated from simulation for all the process corners and the worst possible value needs to be selected thus making the acceleration process slow for all the corners even though not required. This motivates the ongoing work to extend and present a self-learning concept which uses only digital components. The self-learning module is intelligent enough to calculate uncertainty window for all the corners by itself as the SAR ADC operates in conventional mode and use it during acceleration. Therefore self-learning module calculates the digital value of the auxiliary levels by itself whenever in operation.

Self-learning module is made of 3 components, the first component is a set of shift registers and it latch the values of $X_{0} X_{1} X_{2} X_{3} X_{4}$. The second component is an absolute difference calculation circuit that calculates the digital value of the input given to DAC and is of 9-bit size. This is actually done by calculating the difference between the SAR register switching logic value $\left(S_{1} S_{2} \ldots S_{i} \ldots S_{10}\right)$ and the output of ADC $\left(D_{1} D_{2} \ldots D_{i} \ldots D_{10}\right)$. In the SAR logic each bit turns high every cycle starting from MSB bit and its analog value is compared with comparator. If the $i^{\text {th }}$ digit differs in SAR logic and the digital output, the difference is the 2's complement of the digital output code starting from $(i-1)^{\text {th }}$ bit to LSB and the $i^{\text {th }}$ bit is not taken into account, while if the $i^{\text {th }}$ bit is same then the difference is rest of the digital code from $(i-1)^{\text {th }}$ bit to LSB. Note that zero padding is done to

Table 2: Absolute difference calculation example

\begin{tabular}{|c|c|c|}
\hline SAR logic & Digital code & Absolute difference \\
\hline \hline 1000 & 0101 & 0011 \\
\hline 0100 & 0101 & 0001 \\
\hline 0010 & 0101 & 0001 \\
\hline 0001 & 0101 & 0000 \\
\hline
\end{tabular}


the output from the $i^{\text {th }}$ bit to the $9^{\text {th }}$ bit. This is explained in Table 2 for a 4-bit ADC. Also, the carry output of the adder (used for 2's complement calculation) is taken as the MSB bit to have a 10-bit output. If the difference value calculated is actually lower than the lowest auxiliary level, then it is of no use and hence we can stop calculating it. It has to be noted that only one difference value is calculated each cycle. This circuit can work only if we have at least one digital output code available from the SAR ADC.

Finally, the third component of the self-learning module is auxiliary level update memory table constructed using a group of 10-bit registers to store the difference values from the second component. So, each bound of an auxiliary voltage level corresponds to a 10-bit register value. Here we use 4 auxiliary levels in each half of search space, so there will be eight 10-bit registers to store UB and LB values for each of them. Initially, all the registers that store the values for the UB of the auxiliary levels are set to 1 and the LB registers are reset to 0 . A 10-bit register is updated with the values of difference calculation circuit based on $X_{i}$ value that corresponds to a specific auxiliary level. During the update, care should be taken such that if we are updating the registers for the UB of voltage levels, the newly calculated difference value should be larger than the value already stored, while for the registers of LB of voltage levels it has to be lower. So, in order to implement this we need an 11bit subtractor circuit. An exception for the above rule is that the first update always replaces the set and reset values. Self-learning circuit has to be operated long enough to fill this table with values such that the uncertainty window will be smaller for better acceleration. In a way, the proposed self-learning technique will avoid the confusion in selecting auxiliary levels with proper accuracy and also address the comparator offset associated with them. This ends the discussion on self-learning module.

Once the table is created, the next step will be to use these values during the proposed mode. In the proposed mode the UB and LB are updated with the values from this table based on the values of $X_{i}$ from the comparator circuit during the present conversion cycle. The update policies for the UB and LB are shown in Fig. 7. At the time of bound update, if $X_{i}$ is equal to 1 then the UB of $X_{i-1}$ auxiliary level is updated to LB of $X_{i}$ and the LB of $X_{i+1}$ auxiliary level is updated to UB of $X_{i}$ thus internally subtracting or adding uncertainty window as required for update. The new value of $\mathrm{D}$ that has to be given to the $\mathrm{DAC}$ will now be $\frac{U B+L B}{2}$.

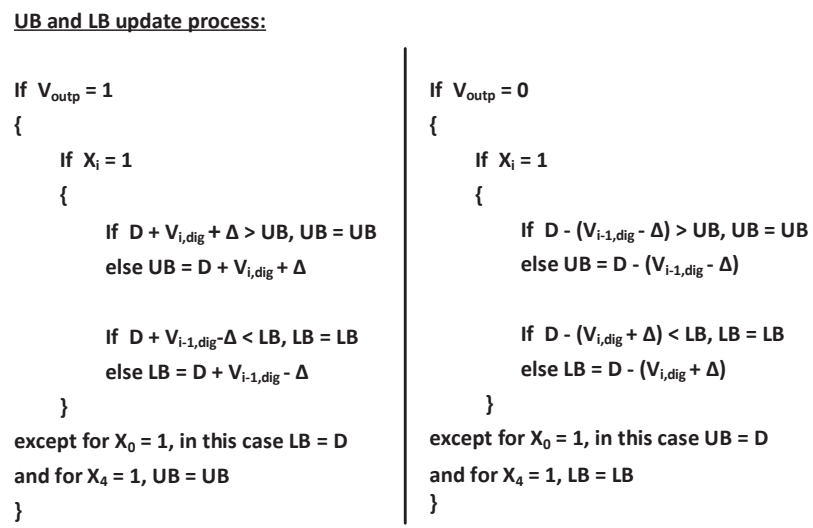

Figure 7: Bound update procedure

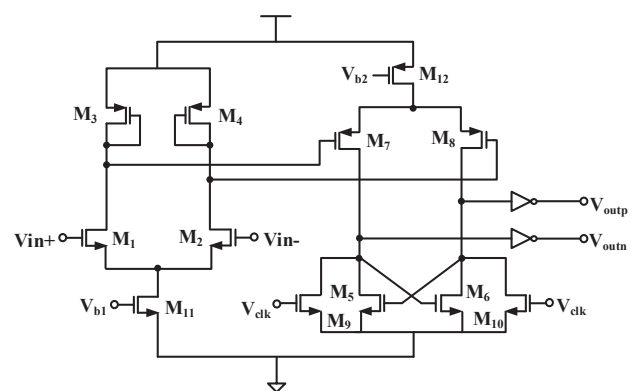

Figure 8: Schematic of Comparator

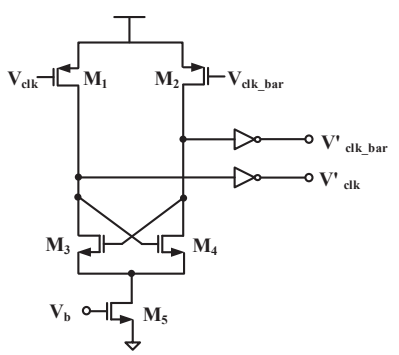

Figure 9: Schematic of special delay element

\section{CIRCUIT IMPLEMENTATION}

The SAR ADC can be configured to work in the conventional mode or the proposed mode using a control signal acceleration-enable. Similarly, self-learning module is also controlled by a self-learning-enable signal. When the circuit is in self-learning phase, ADC continues to function in conventional scheme. The common components of 2 ADC designs will share the same design thus reducing circuit overhead to a certain extent. The auxiliary levels for this prototype design are selected as $3,11,35$ and $73 V_{L S B}$. The design of each component in the ADC is explained as follows:

\subsection{DAC design:}

A split-capacitor based 10-bit CS DAC with differential array is used in this design. The split-capacitive array consists of a pair of MSB and LSB arrays, each of 5-bits, connected by an attenuation capacitor. The unit capacitance is selected as 10fF. Consecutively for the rest of the bits, sizes are increased in power of 2. So, the MSB and LSB array will contain the capacitors of value $10,20,40,80$ and 160 $\mathrm{fF}$. Attenuation capacitor is also chosen as $10 \mathrm{fF}$. Hence, the total amount of capacitance used in DAC is $1260 \mathrm{fF}$.

\subsection{Comparator:}

The schematic of the comparator is shown in Fig. 8. It consists of a pre-amplifier followed by an NMOS cross-coupled latch comparator. During the reset phase $(\mathrm{clk}=1)$ both the outputs of comparator are set to high, and when clk $=0$, the comparator will be put in evaluation phase. Note that there will be some additional components added to this comparator as seen in Fig. 5 required for proposed mode.

\subsection{Comparator delay element:}

Fig. 9 shows the schematic diagram of the comparator delay element required to latch the special XOR gate output. Where $V_{c l k}^{\prime}$ and $V_{c l k \_b a r}^{\prime}$ are delayed outputs with respect to $V_{c l k}$ and $V_{c l k \_b a r}$. The delay element is similar to latch comparator structure and hence follows the comparator decision time in all the process corners theoretically. It has an NMOS 


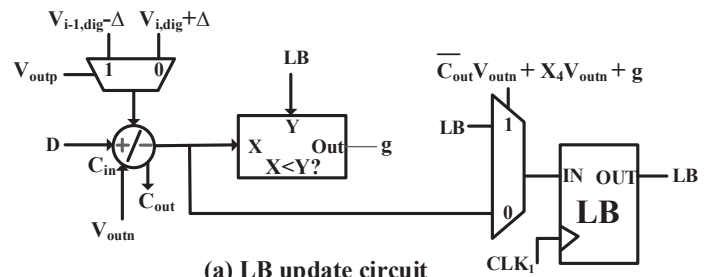

(a) LB update circuit

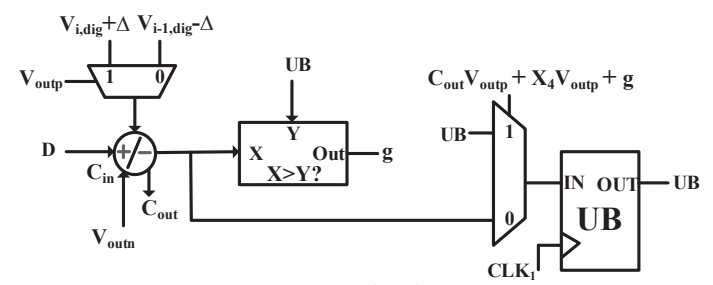

(b) UB update circuit

Figure 10: LB and UB update circuit design

tail current source to control the amount of current flow during the switching of cross coupled NMOS pair as against to the PMOS current source in the latch comparator. The required delay for clock is achieved just by changing the width of the cross coupled NMOS pair.

\subsection{Self-learning module:}

\subsubsection{Set of shift registers}

It contains four set of 8-bit shift-registers to latch $X_{1}, X_{2}$, $X_{3}$ and $X_{4}$ values needed for auxiliary level memory table update. Note that it is not essential to store $X_{0}$ because it can be derived from the remaining values stored and this also reduces the circuit overhead. The lowest auxiliary level selected here is $3 V_{L S B}$ which can be represented by 2-bits. Hence, as discussed previously a 1-bit difference value is of no use and the minimum difference value should be of 2-bits. Because of this reason 8-bit shift registers are used.

\subsubsection{Absolute difference calculator}

Based on its function as explained in Section 2, it takes the digital data of previous cycle and it either forwards the required bits as necessary or provides a 2's complement with the help of a mux. Note that, this module calculates a difference value each cycle with the help of control signals generated from a token shift register used for successive approximation.

\subsubsection{Auxiliary level update memory table}

It has 8 sets of 10-bit registers to store the difference calculator values based on $X_{1}, X_{2}, X_{3}$ and $X_{4}$ values. It also has two 11-bit subtractors and two 10-bit mux for proper update policy as discussed.

\subsection{LB and UB update circuit:}

These circuits are part of proposed SAR ADC. The logic circuits to implement LB and UB update policies are given in Fig. 10. During the update care has to be taken that UB value should decrease and the LB value should rise.

During the proposed mode we need to calculate the SAR voltage level $\mathrm{D}$ as $\frac{U B+L B}{2}$ and a 10 -bit adder is used for this purpose. A mux is used to send appropriate $D$ value during the conventional and proposed mode and also to the $\mathrm{ADC}$ output register. To identify the completion of data conversion in the proposed mode a detection circuit is built

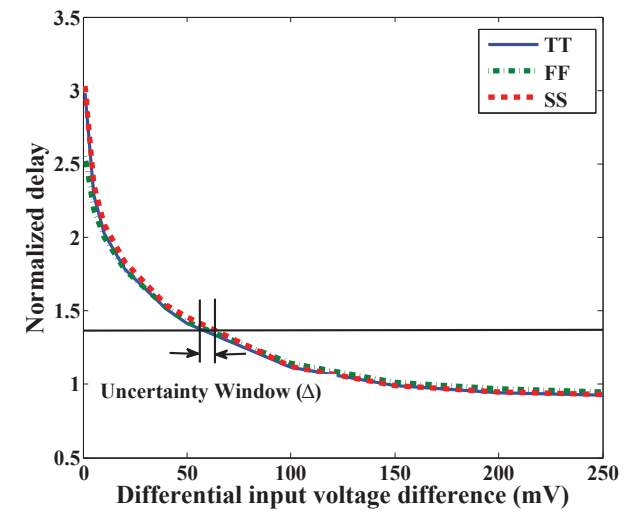

Figure 11: Normalized comparator delay with special delay element

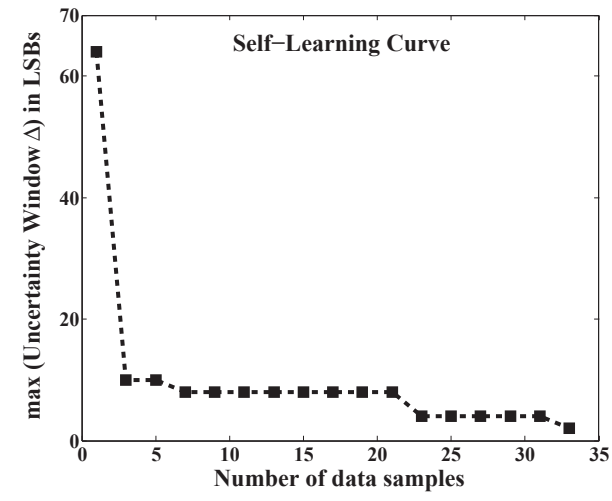

Figure 12: Self-Learning curve

to rise a done signal. Once the done signal goes high all the switching activity is halted till the beginning of next data conversion.

\section{SIMULATION RESULTS}

The proposed acceleration algorithm and the circuit techniques are implemented in a 10-bit SAR ADC using a 130 nm CMOS technology. Both the supply voltage $V_{d d}$ and the reference voltage $V_{\text {ref }}$ are chosen as $0.5 \mathrm{~V}$ and the ADC operates in differential mode. Simulation results in Fig. 11 justify the theoretical assumption of implementing delay element similar to comparator structure as the curves under different process corners converge close enough. However, the uncertainty in selecting auxiliary levels is taken care during bound update procedure. To find out how long we need to operate the self-learning module, a fully differential railto-rail sinusoidal signal is applied at the input. The plot in Fig. 12 shows the amount of learning the circuit has done and it is associated with the reduction in the value of estimated uncertainty window. It can be seen that learning during the first few data points is aggressive and slows down later. Therefore, the longer the time of operation is, the smaller will be the uncertainty window leading to a better acceleration. The trade-off with the above operation is, the lesser the time self-learning module operated the more the number of conversion cycles the proposed SAR ADC takes and vice versa. Hence, it is good to run self-learning module for longer times. Once we construct the auxiliary level update table as required we can turn-off the self-learning module.

Simulation results indicate that both circuit operation models achieve the same accuracy since they use common 


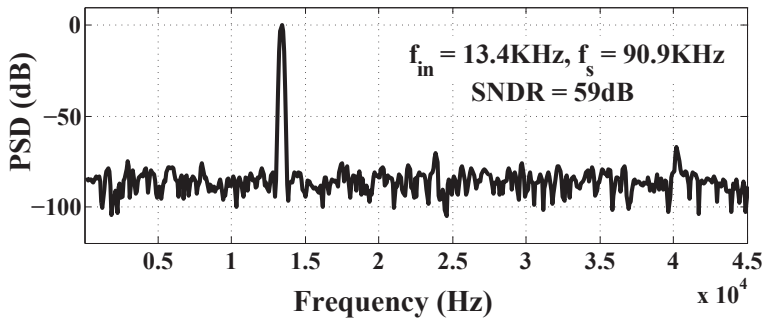

Figure 13: Simulated 1024-point FFT plot for the output

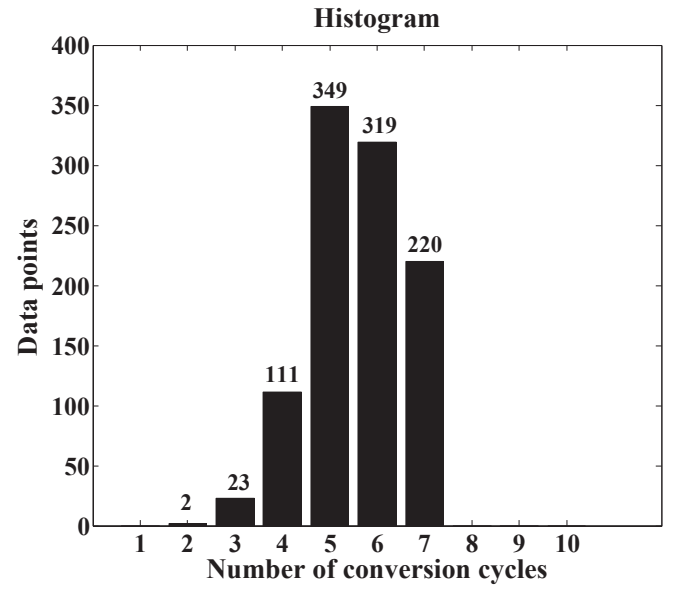

Figure 14: Histogram for 1024 data points

CS DAC as well as comparator. Fig. 13 shows the ADC output spectrum for the proposed technique. In the simulation, the signal frequency is $13.4 \mathrm{KHz}$ and the ADC operates with a $1 \mathrm{MHz}$ clock. The obtained SNDR is approximately $59 \mathrm{~dB}$, corresponding to an ENOB of 9.5-bits. Fig. 14 shows the histogram of number of conversion cycles for 1024 data points in the proposed mode for a maximum uncertainty window of $6 V_{L S B}$. It can be seen that majority of the conversions take 5 or 6 cycles and no conversion takes more than 7 cycles. This shows that the proposed mode is $30 \%$ faster than the conventional mode of operation, which is a significant improvement. Also, the average number of conversion cycles is 5.58 for 1024 data points. The amount of acceleration achieved can be much higher if we can make an optimal selection of auxiliary levels. It should also be noted that the largest auxiliary level that can be selected will be around $100 V_{L S B}$ because of the comparator decision time being almost the same, thus limiting the usage of the proposed acceleration technique. Furthermore, we can also add multiple auxiliary levels as required to improve the conversion speed but it adds to circuit overhead. Hence, the optimal selection of voltage levels needs to be further investigated.

\section{CONCLUSIONS}

In this work a novel time and voltage based SAR ADC technique is presented. The proposed technique enables the use of a single comparator to achieve the acceleration instead of multiple comparators. A new concept of fully digital selflearning technique is also introduced eliminating the need of calculating the worst uncertainty window. Furthermore, the delay elements when implemented in the same fashion as latch comparator allows the circuit to work under differ- ent corners thus making it process independent and robust. To demonstrate the potentials of the proposed techniques, a 10-bit SAR ADC is designed and simulation results show that it takes a maximum of 7 cycles to complete the conversion process. The future research efforts will be directed towards optimizing various aspects of the proposed method, including optimal auxiliary level selection, circuit overhead reduction, etc. It is expected that such optimization will further improve the effectiveness of the proposed techniques.

\section{ACKNOWLEDGEMENTS}

This research has been supported in part by grant NSF IIP 1535658 and the NSF I/UCRC for Embedded Systems at SIUC under grant NSF IIP 1361847. Any opinions, findings, and conclusions or recommendations expressed in this material are those of the authors and do not necessarily reflect the views of the National Science Foundation.

\section{REFERENCES}

[1] Jiayi Jin, Yang Gao, and E. Sanchez-Sinencio. An EnergyEfficient Time-Domain Asynchronous 2 b/Step SAR ADC With a Hybrid R-2R/C-3C DAC Structure. Solid-State Circuits, IEEE Journal of, 49(6):1383-1396, June 2014. ISSN 0018-9200.

[2] Hegong Wei, Chi-Hang Chan, U-Fat Chio, Sai-Weng Sin, Seng-Pan U, R.P. Martins, and F. Maloberti. An 8-b 400MS/s 2-b-Per-Cycle SAR ADC With Resistive DAC. SolidState Circuits, IEEE Journal of, 47(11):2763-2772, Nov 2012. ISSN 0018-9200. .

[3] Hyeok-Ki Hong, Wan Kim, Hyun-Wook Kang, Sun-Jae Park, M. Choi, Ho-Jin Park, and Seung-Tak Ryu. A DecisionError-Tolerant $45 \mathrm{~nm}$ CMOS 7b 1 GS/s Nonbinary 2b/Cycle SAR ADC. Solid-State Circuits, IEEE Journal of, 50(2): 543-555, Feb 2015. ISSN 0018-9200.

[4] M. Rahman, A. Sanyal, and Nan Sun. A Novel Hybrid Radix3/Radix-2 SAR ADC With Fast Convergence and Low Hardware Complexity. Circuits and Systems II: Express Briefs, IEEE Transactions on, 62(5):426-430, May 2015. ISSN 1549-7747.

[5] K. Yoshioka, A. Shikata, R. Sekimoto, T. Kuroda, and H. Ishikuro. An 8 bit $0.3-0.8 \mathrm{~V}$ 0.2-40 MS/s 2-bit/Step SAR ADC With Successively Activated Threshold Configuring Comparators in $40 \mathrm{~nm}$ CMOS. Very Large Scale Integration (VLSI) Systems, IEEE Transactions on, 23(2):356-368, Feb 2015. ISSN 1063-8210.

[6] T. Waho. Non-binary Successive Approximation Analog-toDigital Converters: A Survey. In Multiple-Valued Logic (IS$M V L), 2014$ IEEE 44th International Symposium on, pages 73-78, May 2014.

[7] Chun-Cheng Liu, Soon-Jyh Chang, Guan-Ying Huang, Ying$\mathrm{Zu}$ Lin, Chung-Ming Huang, Chih-Hao Huang, Linkai Bu, and Chih-Chung Tsai. A 10b 100MS/s 1.13mW SAR ADC with binary-scaled error compensation. In Solid-State Circuits Conference Digest of Technical Papers (ISSCC), 2010 IEEE International, pages 386-387, Feb 2010.

[8] A. Shikata, R. Sekimoto, T. Kuroda, and H. Ishikuro. A 0.5 V 1.1 MS/sec 6.3 fJ/Conversion-Step SAR-ADC With TriLevel Comparator in $40 \mathrm{~nm}$ CMOS. Solid-State Circuits, IEEE Journal of, 47(4):1022-1030, April 2012. ISSN 00189200.

[9] Yuan Zhou, Benwei Xu, and Yun Chiu. A 12 bit $160 \mathrm{MS} / \mathrm{s}$ Two-Step SAR ADC With Background Bit-Weight Calibration Using a Time-Domain Proximity Detector. Solid-State Circuits, IEEE Journal of, 50(4):920-931, April 2015. ISSN 0018-9200.

[10] J. Guerber, H. Venkatram, M. Gande, A. Waters, and U. Moon. A 10-b Ternary SAR ADC With Quantization Time Information Utilization. Solid-State Circuits, IEEE Journal of, 47(11):2604-2613, Nov 2012. ISSN 0018-9200. . 